\title{
F-Interop - Online Platform of Interoperability and Performance Tests for the Internet of Things
}

\footnotetext{
Sébastien Ziegler' ${ }^{1}$ Loïc Baron², Brecht Vermeulen ${ }^{3}$ and Serge Fdida ${ }^{2}$

${ }^{1}$ Mandat International, Switzerland

${ }^{2}$ University Pierre \& Marie Curie LIP6, France

${ }^{3}$ IMEC, Belgium

\subsection{Introduction}

The Internet of Things (IoT) will be massive and pervasive, with 50 to 100 Billion smart things and objects connected by 2020. It will impact diverse application domains, from smart cities to agriculture, from manufacturing to eHealth and energy. The success of this new technological revolution will require adequate standardization and interoperability. Since 1995, interoperability is recognized by the International Telecommunication Union (ITU) as the main obstacle to IoT development and adoption by the market.

F-Interop (www.f-interop.eu) [1] is a European research project addressing this challenge, by researching and developing an online platform of testing tools for the IoT, including:

- Interoperability tests

- Conformance tests

- Performance tests, including scalability, Quality of Service, and Privacy.

It intends to support and accelerate the development of emerging IoT standards and once the standard is stable enough to support SMEs to align and comply with such standards.
} 


\subsection{Context and Problematic}

In order to be widely adopted, new technologies, products and solutions go through several steps:

- Standardization: stakeholders discuss and align their views to converge towards common standards and specifications.

- Conformance \& Interoperability: stakeholders test and validate that their implementation is conform to the standard.

- Optimization: in terms of Quality of Service, scalability, energy consumption, etc.

- Market Launch: the solution is ready for roll-out into the market.

Each phase traditionally requires extensive testing, where different vendors meet face-to-face to test interoperability by going through an exhaustive list of "interoperability tests". The consequence is that:

- The current process is extremely labor-intensive, as engineers travel across the globe often only to find out what they need to make a minor fix;

- The cost associated with engineering time and travel expenses is often too high for SMEs;

- Time-to-market is unnecessarily stretched, giving vendors who want to adopt emerging standards a disadvantage compared to vendors who come to market with entirely proprietary solutions.

The concept of F-Interop is somehow to "dematerialize" the process of testing, exploiting the asset of the European FIRE research infrastructure. It aims to develop online and remote interoperability and performance test tools supporting emerging technologies from research to standardization and to market launch. The outcome will be a set of tools enabling:

- Standardization communities to save time and resources, to be more inclusive with partners who cannot afford travelling, and to accelerate standardization processes;

- SMEs and companies to develop standards-based interoperable products with a time-to-market cut by 6-12 months, and significantly lowered engineering/financial overhead.

\subsection{Technical Approach and Outcomes}

The goal of F-Interop is to extend FIRE+ with online interoperability and performance test tools supporting emerging IoT-related technologies from research to standardization and to market launch for the benefit of researchers, 
product development by SMEs, and standardization processes. Specifically, F-Interop will combine three complementary approaches:

\subsubsection{Online Testing Tools}

First and foremost, F-Interop is researching and developing online testing tools for the IoT, enabling to test interoperability, conformance, scalability, Quality of Service (QoS), the Quality of Experience (QoE), and energy efficiency of IoT devices and services.

Testbeds federations with a shared "Testbed as a Service"

F-Interop brings together 3 testbed federations and facilities, encompassing over 32 testbeds and thousands of IoT nodes, with:

- Fed4FIRE, which federates 24 FIRE+ testbeds, bringing together cloud, IoT, wireless, wireless mobile, LTE, cognitive radio, 5G, openflow, SDN, NFV and network emulation technologies.

- OneLab, which federates testbeds for the future Internet, including IoT, cognitive radio, wireless, cloud and overlay network technologies.

- IoT Lab, which federates IoT and crowdsourcing/crowd-sensing testbeds, including smart campus, smart building and smart office testbeds.

In order to support this integration, F-Interop is extending the testbeds federation architecture model with a new layer enabling shared services among several testbed federations. This approach enables to interface "Testbed as a Service" (TBaaS) with existing federations through a clearly specified API, enabling remote access and interaction with various experimental platforms.

As we can see, Fed4Fire, OneLab and IoT Lab were mostly providing access to raw resources (compute, storage, network, data). F-Interop will propose access to a higher, richer service focused on IoT testing and exploiting the resources of the federated underlying testbeds.

\subsubsection{Support and to loT Standardization and Industry}

F-Interop works in close collaboration with several standardization bodies, and is directly contributing to three IoT standardization processes: oneM2M, 6TiSCH (IETF) and the Web of Things (W3C). It will also explore the possibility to support and enable new online certification and labelling mechanisms such as the IPv6 Ready logo. More generally, F-Interop intends to enable an easier participation of researchers and industry in standardization processes. 


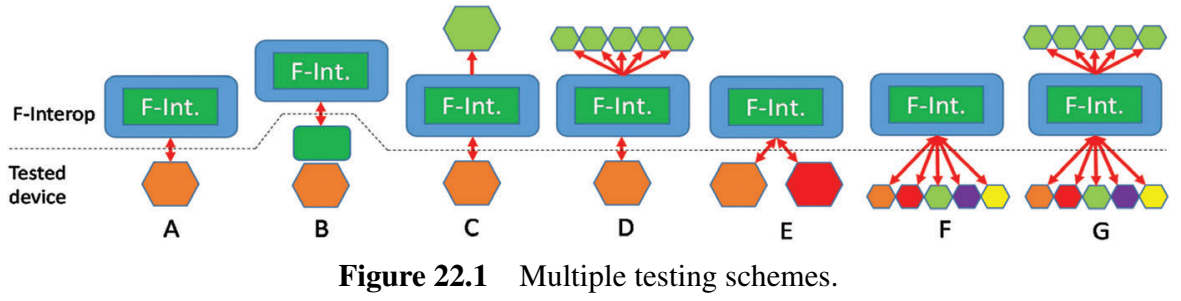

It will also run an open call for SMEs and developers, inviting them to use and enrich the platform with additional modules and extensions.

\subsubsection{Flexible Testing Schemes}

F-Interop is researching and exploring various testing schemes and configurations, by interconnecting devices under tests with the server testing tools, resources provided by the F-Interop connected federations of testbeds, and resources provided by other users, as illustrated in Figure 22.1, where the salmon hexagon represents a device under test.

A. Tested Device using F-Interop Platform

B. Deported test with downloaded resource

C. Remote interop with 2 participants

D. Interop against testbed

E. Local interop with 2 participants

F. Remote interop with $\mathrm{N}$ participants

G. Remote interop with $\mathrm{N}$ participants and testbeds

\subsection{Architectural View}

\subsubsection{F-Interop Platform and Test Tools}

F-Interop offers a service allowing users to plug an IoT implementation and run interoperability, conformance and performance tests. The implementation under test can be a device owned by a user but it can also be hosted at a testbed among the three federations having a partnership with F-Interop: Fed4FIRE, OneLab and IoT Lab. This service is composed of the following building blocks:

- Implementations under test.

- Testbed federations (hosting implementations and the F-Interop service itself). 


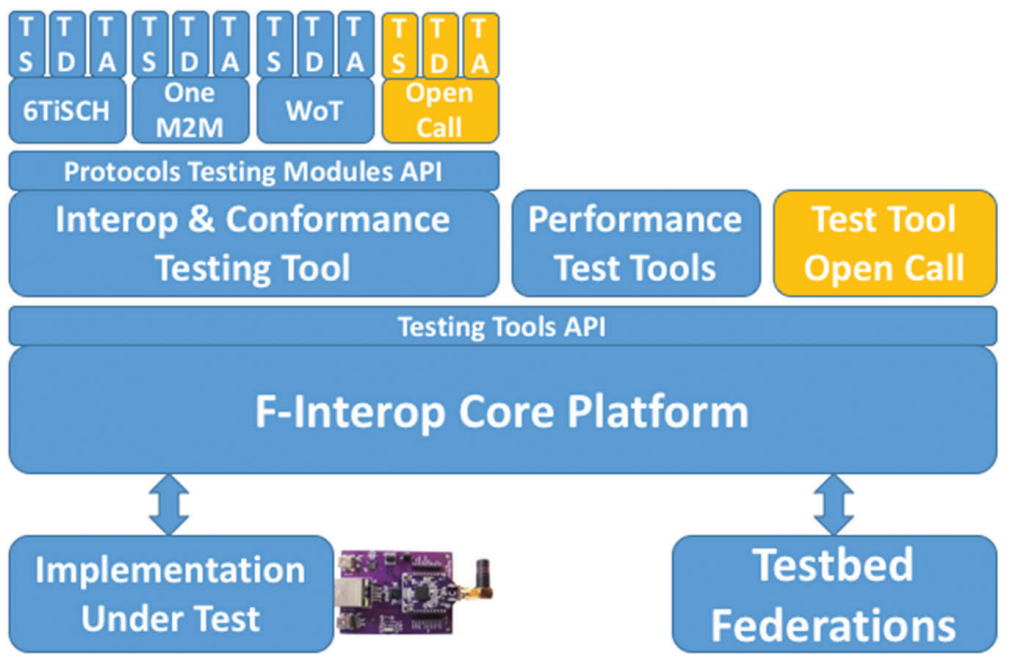

- F-Interop Core Platform is a bus of events ensuring the exchange of messages between devices and test tools.

- Test tools are responsible for orchestrating the execution of the tests.

- The tests must be defined in three steps: test specification (TS), test description (TD) and test analyzer (TD).

- TS: test specification

- TD: test description

- TA: test analyzer issues verdicts about the results of the tests.

\subsubsection{F-Interop Architecture}

The basic architecture of F-interop answers three requirements:

- Allowing users to do remote interop, conformance and performance testing.

- Allow users to use devices in testbeds for this testing (varying from using only testbed resources such as IoT sensors, virtual machines, etc, to combining resources at the user premises with the testbed resources).

- Allow contributors to extend the F-interop framework with extra functionality and tests.

The figure below shows the basic architecture with two different users doing interop/conformance testing against the F-interop central servers. The graphical user interface (GUI) is the interaction point with the F-interop system (creating account, logging in, choosing test, start test, see results of tests). 
The GUI talks to the orchestration and analysis engine to start, stop, analyze tests, while the orchestration engine talks to the F-interop agent running nearby the devices under test (e.g. on a laptop connected with USB to the IoT devices). All communication is done through secured AMQP messaging. After testing, results are stored in a central result repository (where a user can access his own results or give access to other people on demand). The resource repository lists all available devices (in testbeds and at user's premises who want to give remote access to their devices for testing).

The figure depicts two different users on two different locations, but of course these can be two users on the same location, or a single user, depending on the exact test.

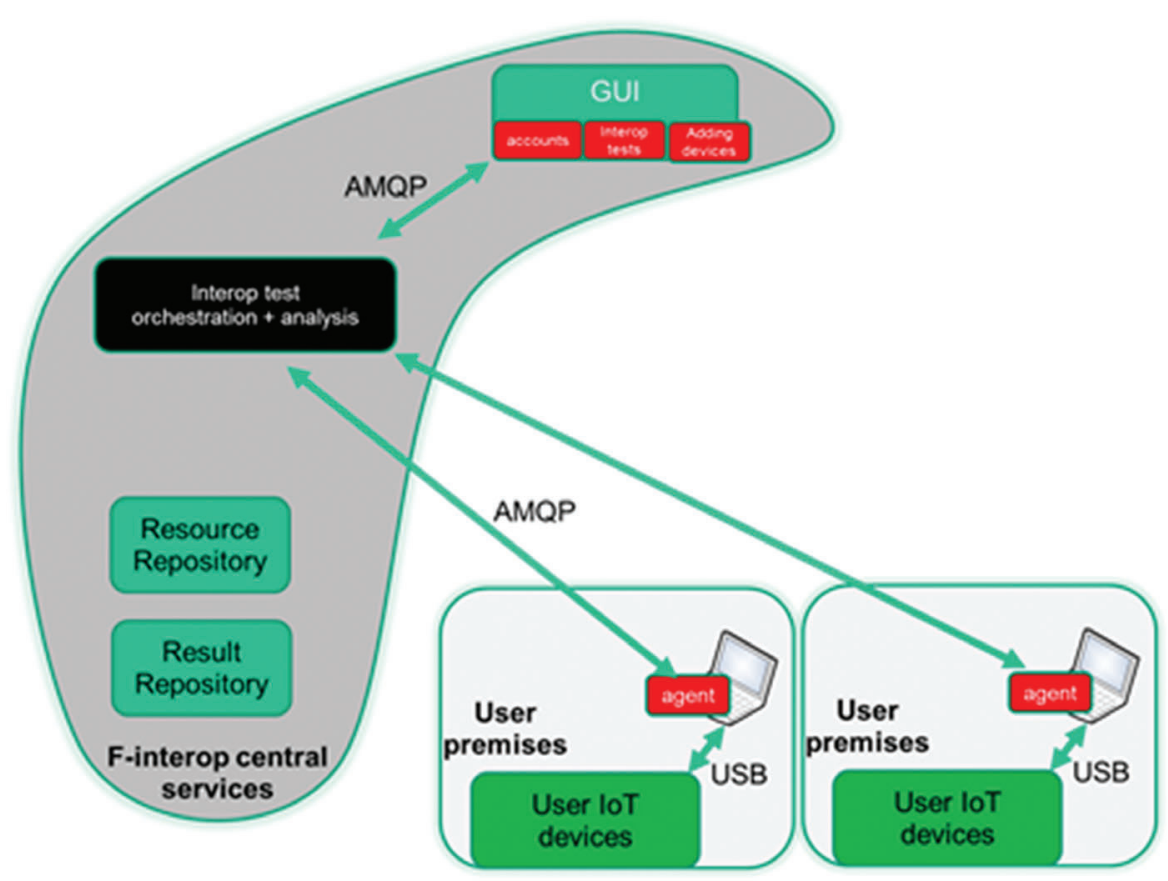

The next figure shows a similar architecture, but now resources at testbeds are involved. The figure depicts an example where a user tests devices at his premises against devices at a testbed, but of course alternatives such as running everything on a testbed, combining resources at multiple testbeds, etc are all possible. The components are merely the same, but as can be seen, there is now a TestBed as a Service Layer (TBaaS) which reserves and provisions 
resources at testbeds and then launches the test suites. Interesting here is that also the orchestration and analysis modules run on virtual machines on testbed and allow full access for users. This makes it possible for contributors of test and analysis software to deploy their own instantiation of part of the F-interop platform and as such they can extend it easily and contribute back to F-interop.

On top of the TBaaS layer, there is also an external API based on REST and based on the testbed standard resource description called an RSpec (XML based). A full blown test can be launched easily in this way.

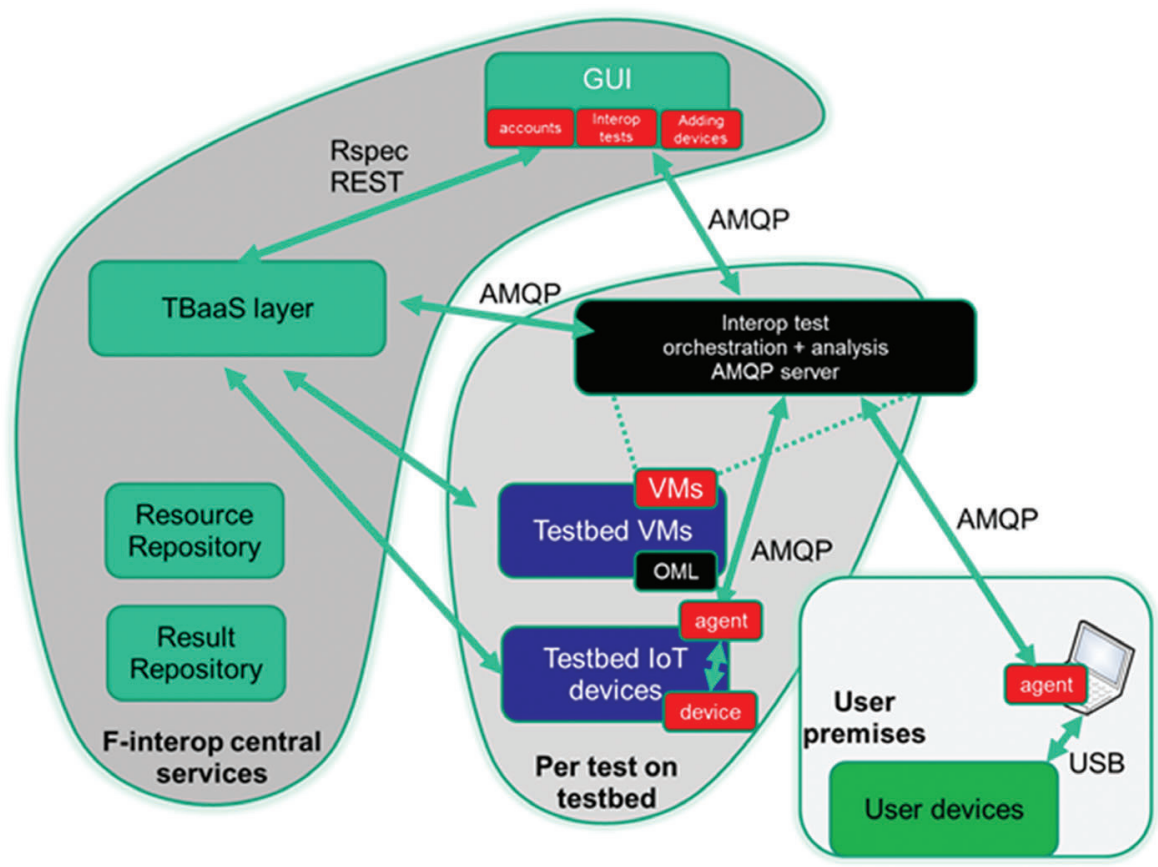

\subsection{Open Call}

F-Interop is already developing tools supporting a set of core protocols (e.g. 6TiSCH, One M2M, CoAP) and defining standard APIs to allow easy plug and extension with new tools and protocols. This will allow to inject events in the network in order to test protocols performance under different conditions and identify possible intervention points for modifications and improvements of standards. 
To further growth the ecosystem of protocols, communities and devices the F-interop platform can support, thus cutting the time for interoperability tests of businesses operating in the IoT space, the project is now organising competitions to partner with external businesses (with funding ranging from $10 \mathrm{~K}$ to $100 \mathrm{~K}$ EUR depending on project nature) to extend the core platform with new tools and to exploit the current open nature of the designed architecture and APIs.

We are currently planning dissemination and communities engagement activities, in order to promote the project aims and to engage with third parties interested to apply for funding and to understand the possible tools that the platform might benefit from.

The timeline is provided below.

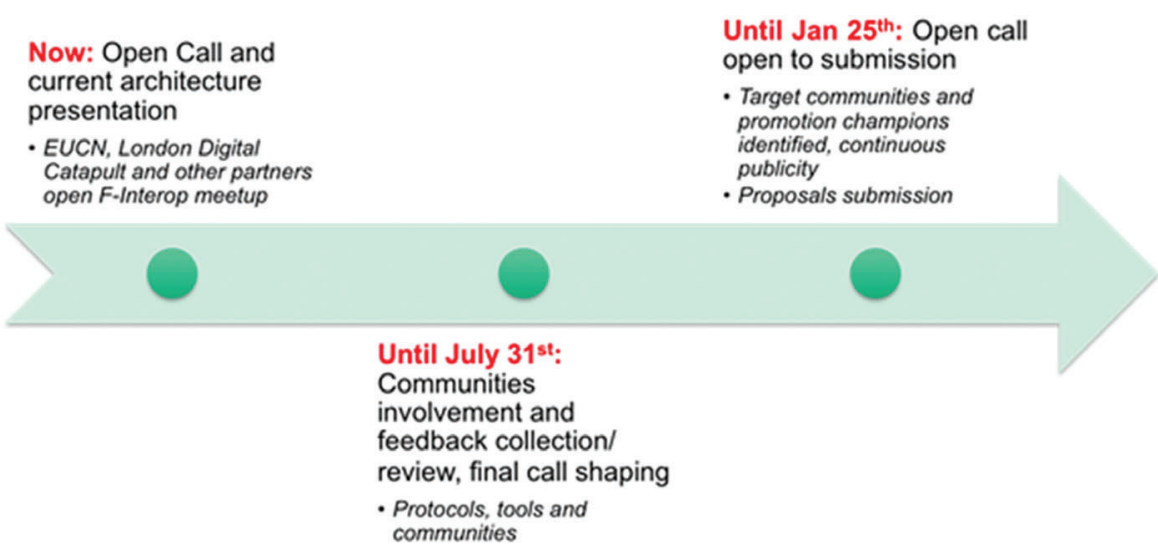

More details on the open call and provided grants can be found here: http://www.f-interop.eu/index.php/open-call

If you are involved with one of the following communities (BLE, OMA LWM2M, 6TiSCH, CoAP/6LowPAN/RPL, IPv6 ready, Low Power Wide Area Network, Web of Things, Hypercat, COEL, 5G IoT) or new emerging ones (e.g. Internet of Underwater Things) in the IoT ecosystem, you might be interested in the F-interop open call.

\subsection{Conclusion}

F-Interop is a new project that strongly changes the potential usage of the FIRE testbeds. Indeed, the principle is to provide remote testing, conformance and performance test exploiting the large set of resources exposed 
by the underlying testbeds or federation of testbeds. In order to achieve this goal, F-Interop is developing architecture for TaaS as well as an adequate APIs to bridge the gap between what exists today, serving general-purpose experiments and our target related to IoT interoperability testing. Therefore, we can see that F-Interop will bring together two communities (FIRE and Test community) and expect to impact the way testing is done today, especially in the growing field of IoT where heterogeneity is key. 
